Supporting Information

\title{
Jones matrix microscopy for living eukaryotic cells
}

Kwanjun Park, ${ }^{1}$ Taeseok Daniel Yang, ${ }^{1,2}$ Dongkwon Seo, ${ }^{1,3}$ Min Gyu Hyeon, ${ }^{1}$ Taedong Kong, ${ }^{1}$

Beop-Min Kim, ${ }^{1,3}$ Yeonho Choi, ${ }^{1,3}$ Wonshik Choi, ${ }^{4,5}$ and Youngwoon Choi ${ }^{1,3^{*}}$

${ }^{1}$ Department of Bioengineering, Korea University, Seoul 02841, South Korea

${ }^{2}$ School of Biomedical Engineering, Brown University, Providence, Rhode Island 02912, USA

${ }^{3}$ Interdisciplinary program in Precision Public Health, Korea University, Seoul 02841, South Korea

${ }^{4}$ Center for Molecular Spectroscopy and Dynamics, Institute for Basic Science (IBS), Seoul 02841, South

Korea

${ }^{5}$ Department of Physics, Korea University, Seoul 02841, South Korea

*Correspondence: youngwoon@korea.ac.kr 


\section{Supporting Information 1: Experimental setup}

The experimental setup for the polarization phase microscope is schematically presented in Fig. S1 (a). The optical configuration is based on our previous implementation equipped with the capability of the single-shot Jones matrix measurement of a sample ${ }^{1}$. Two He-Ne lasers (S1 and S2; Thorlabs, HNL210L, $\lambda=632.8 \mathrm{~nm}$ ) introduce two orthogonally polarized light waves. The two separate lasers are employed to avoid the unwanted cross-interference between the two waves having orthogonal polarization. After the polarizing beam splitters (PBS1 and PBS2), the two beams are spatially overlapped and co-propagate along the sample path with polarizations orthogonal to each other. In the sample path, the beams are first expanded by a beam expander $(\mathrm{BE} 1 ; 10 \times)$, and then steered by two galvanometer mirrors (GMx and GMy; Thorlabs: GVS211) placed at the planes conjugated to the sample plane. The beams are further delivered by a $4-f$ telescope consisting of a
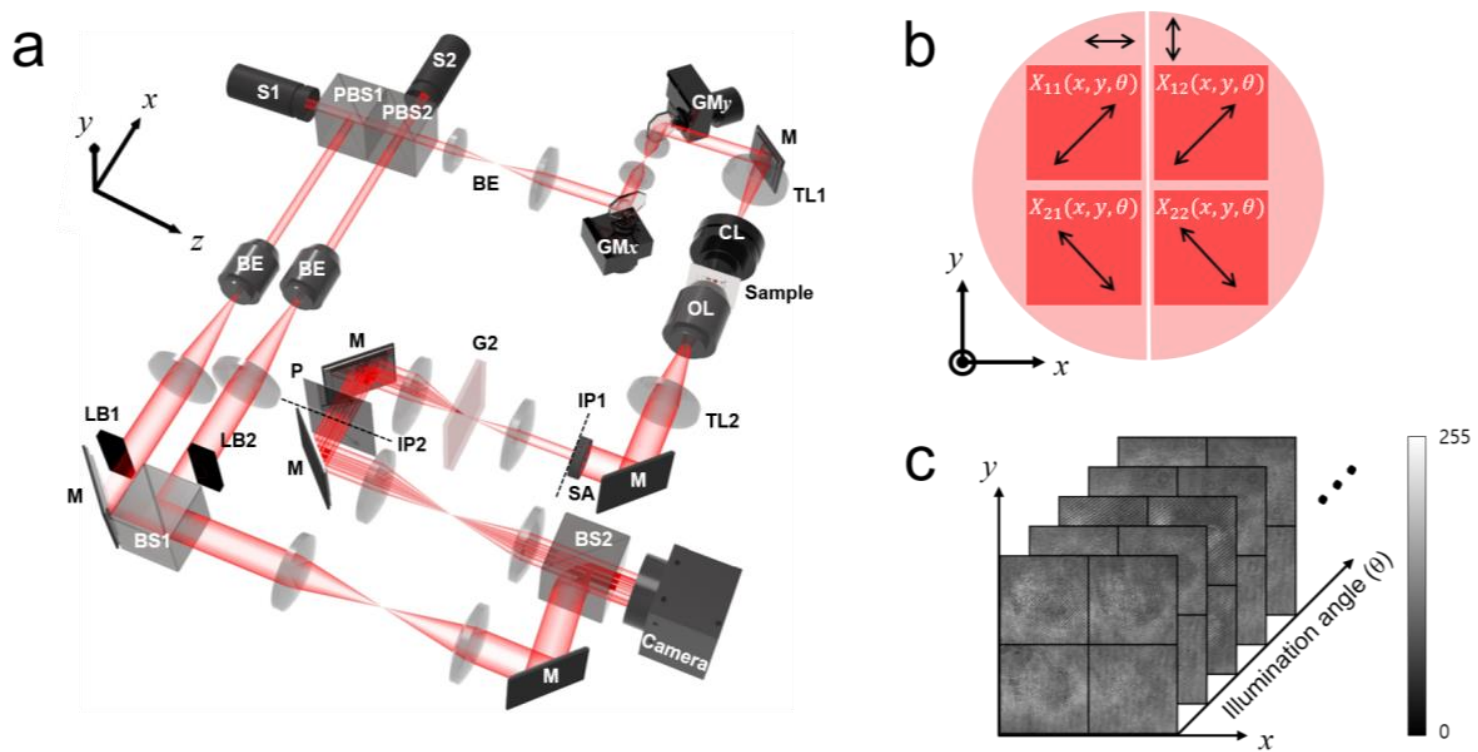

Fig. S1. Experimental schematic for the synthesized Jones matrix microscope system. (a) Schematic diagram of the system. S1 and S2: He-Ne laser sources $(\lambda=632.8 \mathrm{~nm})$, PBS1 and PBS2: polarizing beam splitters, BS1 and BS2: beam splitters, BE: beam expanders, GM $x$ and GMy: galvanometer mirrors, CL: condenser lens, OL: objective lens, TL1 and TL2: tube lenses, M: mirrors, LB1 and LB2: light blocks, SA: square aperture, G2: 2-D diffraction grating, $\mathrm{P}$ : linear polarizers with polarizing axis of $+45^{\circ}$ (upper part), $-45^{\circ}$ (lower part). (b) Polarization configuration in the array of $2 \times 2$ sample images and the reference beam. Bidirectional arrow for each area represents a polarizing axis of the beam in the camera plane. (c) A set of $2 \times 2$ interferograms taken at different illumination angles. 
tube lens (TL1) and a condenser lens (CL; Nikon, 1.4 NA, oil). Thus, the illumination angle is adjusted by the two mirrors with the stationary pivot point on the sample plane.

After transmitting the sample, the image is delivered to an image plane (IP1) through an objective lens (OL; Olympus, 100×, 1.4 NA, oil) and a tube lens (TL2). At the IP1, a square aperture (SA) is placed to trim the observable field of view (FOV). The squared image is delivered through another 4- $f$ telescope to the next image plane (IP2). During the delivery, multiple copies of the squared image are generated by a 2-D grating (G2, two 1-D gratings, Edmund Optics, 80 lines $/ \mathrm{mm}$ ) placed in the Fourier plane of the telescope. Among the 9 copies $(3 \times 3)$, only four images $(2 \times 2)$ generated by 0 -th and +1 -st diffraction orders are selected for imaging by another square aperture (not shown in the figure) placed at the IP2. Since the gratings have almost equal power among the 0-th and \pm 1 -st diffraction orders, the four image copies have the similar level of brightness. The $2 \times 2$ images pass through a composite of two linear polarizers (P; Thorlabs, LPVIS2X2) placed near at IP2. The upper $1 \times 2$ images transmit through a polarizer with +45 -degree polarization axis, whereas the lower $1 \times 2$ images a polarizer with -45 -degree axis as shown in Fig. S1 (b). The $2 \times 2$ images are further delivered to a CMOS camera (Vieworks, VC-12MX-M180) for image acquisition via the next 4- $f$ telescope together with a beam splitter (BS2).

The reference beam also consists of the two orthogonally polarized laser beams split by PBS1 and PBS2. These beams are magnified by beam expanders (BE2, 20x) and a half of each is blocked by a beam block (LB1 or LB2). The two semicircular beams meet at a beam splitter (BS1) and form a single circular beam with no spatial overlap, where the right half is occupied by the light from S1 with vertical polarization and the left half from S2 with horizontal polarization as shown in Fig. S1 (b). The beam propagates further and meets the $2 \times 2$ sample images at the camera via BS2 with the off-axis configuration. Due to the polarization configuration of the four images and the reference beam, each interferogram carries different polarization response of the sample. The camera takes the four interferograms at a single exposure at a single illumination angle. For the synthesis of the 
Jones matrix, multiple $2 \times 2$ images are measured with the scanning of the illumination angle as shown in Fig. S1 (c).

\section{Supporting Information 2: Construction of synthesized Jones matrix}

At a certain illumination angle, a Jones matrix of the sample is constructed from the four subimages taken at a single exposure. A single phase image is known to be prone to the phase noise caused by unwanted diffraction or multiple reflections which may occur at the optics placed in the beam pathways. To suppress the phase noise, the synthetic aperture microscopic technique is employed. The construction of the low-noise Jones matrix is composed of two steps: extracting single Jones matrices from the sets of sub-images acquired at various illumination angles and synthesizing the apertures of the extracted Jones matrices.

Since the extraction procedures for the Jones matrix of a sample from a single set of four subimages are well described in our previous study ${ }^{1}$ and elsewhere ${ }^{2-6}$, here we briefly present the procedures for Jones matrix construction. Assume that the Jones matrix $\mathbf{J}$ of an objective is described by a $2 \times 2$ matrix of a form of $\left(\begin{array}{ll}J_{11} & J_{12} \\ J_{21} & J_{22}\end{array}\right)$, then the sub-images at the camera are formed as

$$
\mathbf{X}=\left(\begin{array}{ll}
\left(J_{11}+J_{21}\right) & \left(J_{22}-J_{12}\right) \\
\left(J_{11}-J_{21}\right) & \left(J_{22}+J_{12}\right)
\end{array}\right)
$$

where $\mathbf{X}$ in Eq. (S1) is the $2 \times 2$ matrix representing the measured complex images of the form $\left(\begin{array}{ll}X_{11} & X_{12} \\ X_{21} & X_{22}\end{array}\right)$. Thus, the extraction of the Jones matrix of the sample is given by the following simple linear algebra as 


$$
\mathbf{J}=\left(\begin{array}{ll}
\left(X_{11}+X_{21}\right) & \left(X_{22}-X_{12}\right) \\
\left(X_{11}-X_{21}\right) & \left(X_{22}+X_{12}\right)
\end{array}\right)
$$

For the aperture synthesis of the Jones matrix, it is required to acquire a set of multiple images taken at various illumination angles. A single Jones matrix image obtained at a specific illumination angle $\left(\theta_{x}^{i l l}, \theta_{y}^{i l l}\right)$ is represented by

$$
\mathbf{J}\left(x, y ; \theta_{x}^{\text {ill }}, \theta_{y}^{\text {ill }}\right)=\left(\begin{array}{cc}
J_{11}\left(x, y ; \theta_{x}^{\text {ill }}, \theta_{y}^{\text {ill }}\right) & J_{12}\left(x, y ; \theta_{x}^{\text {ill }}, \theta_{y}^{\text {ill }}\right) \\
J_{21}\left(x, y ; \theta_{x}^{\text {ill }}, \theta_{y}^{\text {ill }}\right) & J_{22}\left(x, y ; \theta_{x}^{\text {ill }}, \theta_{y}^{\text {ill }}\right)
\end{array}\right)
$$

where $\mathrm{x}$ and $\mathrm{y}$ are the spatial coordinates of the image.

The image synthesis is performed for each element of the Jones matrix in Eq. (S3). The Fourier transform of an element in the Jones matrix can be expressed as

$$
\tilde{J}_{i j}\left(k_{x}, k_{y} ; k_{x}^{i l l}, k_{y}^{i l l}\right)=P\left(k_{x}, k_{y}\right) \tilde{J}_{i j}^{O}\left(k_{x}-k_{x}^{\text {ill }}, k_{y}-k_{y}^{i l l}\right),
$$

where $\tilde{J}_{i j}\left(k_{x}, k_{y} ; k_{x}^{\text {ill }}, k_{y}^{\text {ill }}\right)$ is the Fourier transform of the matrix element $J_{i j}\left(x, y ; \theta_{x}^{\text {ill }}, \theta_{y}^{\text {ill }}\right)$ in Eq. (S3), and $\tilde{J}_{i j}^{O}\left(k_{x}-k_{x}^{\text {ill }}, k_{y}-k_{y}^{\text {ill }}\right)$ is the Jones matrix spectrum of the object shifted by the illumination wavevector $\left(k_{x}^{i l l}, k_{y}^{\text {ill }}\right)$ according to the scanning angle $\left(\theta_{x}^{\text {ill }}, \theta_{y}^{\text {ill }}\right), i$ and $j$ denote the row and column indices in the Jones matrix, either 1 or $2 . P\left(k_{x}, k_{y}\right)$ is the pupil function determined by the NA of the objective lens, which is expressed as

$$
P\left(k_{x}, k_{y}\right)=\left\{\begin{array}{cc}
1, & \sqrt{k_{x}^{2}+k_{y}^{2}} \leq k_{0} \cdot \mathrm{NA} \\
0, & \text { others }
\end{array},\right.
$$

where $k_{0}=2 \pi / \lambda$ with the wavelength of the light $\lambda$.

To recover the unshifted object spectrum in the Jones matrix, the spectrum is shifted back in the Fourier space by $\left(+k_{x}^{\text {ill }},+k_{y}^{\text {ill }}\right)$, yielding $\tilde{J}_{i j}\left(k_{x}+k_{x}^{\text {ill }}, k_{y}+k_{y}^{\text {ill }} ; k_{x}^{\text {ill }}, k_{y}^{\text {ill }}\right)=P_{i j}\left(k_{x}+k_{x}^{\text {ill }}, k_{y}+k_{y}^{\text {ill }}\right) \tilde{J}_{i j}^{O}\left(k_{x}, k_{y}\right)$. The image synthesis is then performed by addition of the individual unshifted object spectrums as 


$$
\begin{aligned}
\tilde{J}_{i j}^{\text {syn }}\left(k_{x}, k_{y}\right) & =\sum_{k_{x}^{\text {ill }}, \mathrm{k}_{y}^{\text {ill }}} \tilde{J}_{i j}\left(k_{x}+k_{x}^{\text {ill }}, k_{y}+k_{y}^{\text {ill }} ; k_{x}^{\text {ill }}, k_{y}^{\text {ill }}\right) \\
& =\sum_{k_{x}^{\text {ill }}, k_{y}^{\text {ill }}} P_{i j}\left(k_{x}+k_{x}^{\text {ill }}, k_{y}+k_{y}^{i l l}\right) \tilde{J}_{i j}^{O}\left(k_{x}, k_{y}\right) . \\
& =\tilde{J}_{i j}^{O}\left(k_{x}, k_{y}\right) \sum_{k_{x}^{i l l}, k_{y}^{i l l}} P_{i j}\left(k_{x}+k_{x}^{i l l}, k_{y}+k_{y}^{i l l}\right)
\end{aligned}
$$

The synthesized Jones matrix element $J_{i j}^{s y n}(x, y)$ is then obtained by taking the inverse Fourier

transform of $\tilde{J}_{i j}^{\text {syn }}\left(k_{x}, k_{y}\right)$. Since the images are accumulated with many illumination angles, the diffraction noise occurring in the Jones matrix images is effectively suppressed ${ }^{7,8}$. Also, due to the addition of the pupil function with a number of shifts in Eq. (S6), the synthesized spectrum of the Jones matrix has the enlarged pupil function beyond the physical limit of the objective NA. This causes the resolution enhancement depending on the angular coverage of the illumination scanning 9 , 10.

To construct a synthesized Jones matrix in the demonstration, typically 100 images are acquired while scanning the two galvanometer mirrors for 0.66 seconds. The scanning range covers $0.8 \mathrm{NA}$ of the condenser lens (full NA $=1.4$ ) to achieve the improved SNR and spatial resolution, but with no significant uncertainty in the birefringence measurements. The acquisition rate is $150 \mathrm{fps}$, which is the limit set by the camera speed.

\section{Supporting Information 3: Effect of angle scanning on polarization measurement}

In our experimental scheme, multiple images are acquired at various illumination angles for synthesizing the Jones matrix. During the illumination scanning, the angle of input light relative to an optic axis of a sample is varied. Since the polarization response is an axis-sensitive phenomenon, the angle steering can cause a different polarization response at each illumination. Thus synthesizing the Jones matrices may result in a certain error in the polarization measurement. In 
this section, we will address the effect of angle scanning on the polarization measurement and estimate the amount of error.

In general case, the electric flux density $\mathbf{D}$ is connected with the electric field $\mathbf{E}$ as $\mathbf{D}=\boldsymbol{\varepsilon E}$, and this can be written in components as $D_{i}=\sum_{j} \varepsilon_{i j} E_{j}$, where $\varepsilon_{i j}$ is the electric permittivity which is a symmetric and second-rank tensor and $i, j=1,2,3$ for $x, y$, and $z$ components, respectively. In a geometrical representation, $\varepsilon_{i j}$ becomes a quadratic surface known as an ellipsoid as $\sum_{i j} \varepsilon_{i j} x_{i} x_{j}=1$ with $x_{1,2,3}$ indicating $x, y$, and $z$.

Although the elements $\boldsymbol{\varepsilon}$ depend on the choice of the coordinate system, but the principal axes can always be found such that $\varepsilon_{i j}=\delta_{i j} \varepsilon_{i}$, where $\delta_{i j}$ is the Kronecker delta. Along the principal axes, $\mathbf{D}$ and $\mathbf{E}$ are parallel to each other and the off-diagonal contributions all vanish. For the simple representation, we choose the coordinate system $x, y$, and $z$ are assumed to be along the material's principal axes. The material is then characterized by the principal refractive indices as $n_{1}=\left(\varepsilon_{1} / \varepsilon_{0}\right)^{1 / 2}, n_{2}=\left(\varepsilon_{2} / \varepsilon_{0}\right)^{1 / 2}$, and $n_{3}=\left(\varepsilon_{3} / \varepsilon_{0}\right)^{1 / 2}$ along the $x, y$, and $z$ axes, respectively, with $\varepsilon_{0}$ the permittivity of free space. Furthermore, with the choice of the principal axes, the geometric representation of the ellipsoid reduces to a simple form as $\varepsilon_{1} x^{2}+\varepsilon_{2} y^{2}+\varepsilon_{3} z^{2}=1$. By combining this with the principal indices above results in so-called the index ellipsoid as

$$
\frac{x^{2}}{n_{1}^{2}}+\frac{y^{2}}{n_{2}^{2}}+\frac{z^{2}}{n_{3}^{2}}=1 .
$$

The optical properties of the material are then described by the refractive index ellipsoid.

\section{Uniaxial Case:}

As a simple case, we will discuss the uniaxial case where two of the principal refractive indices are the same due to a certain symmetry of the material. Let us assume that $n_{1}=n_{2}=n_{o}$, and $n_{3}=n_{e}$ (in this context, $n_{o}$ and $n_{e}$ are the ordinary and extraordinary indices, respectively). Then the 
index ellipsoid becomes an ellipsoid of revolution as shown in Fig. S2 (a). Assume that a light wave propagates through this material along the direction of the wavevector $\vec{k}$. The unit vector $\hat{k}$ along the wavevector has the azimuthal and elevation angles $\alpha$ and $\beta$ with respect to the $z$-axis. Then the light suffers from two normal mode refractive indices $n_{a}$ and $n_{b}$ along the two orthogonal directions. The normal mode directions correspond to two semi-axes of an ellipse which is determined as the intersection of the ellipsoid of revolution and a plane passing through the origin, normal to $\hat{k}$, as shown in Fig. S2 (a). Due to the symmetry along the $x$-axis, one of the normal mode indices is $n_{o}$ regardless of the rotation angle $\alpha$. We assign $n_{o}$ as $n_{b}=n_{o}$ as represented
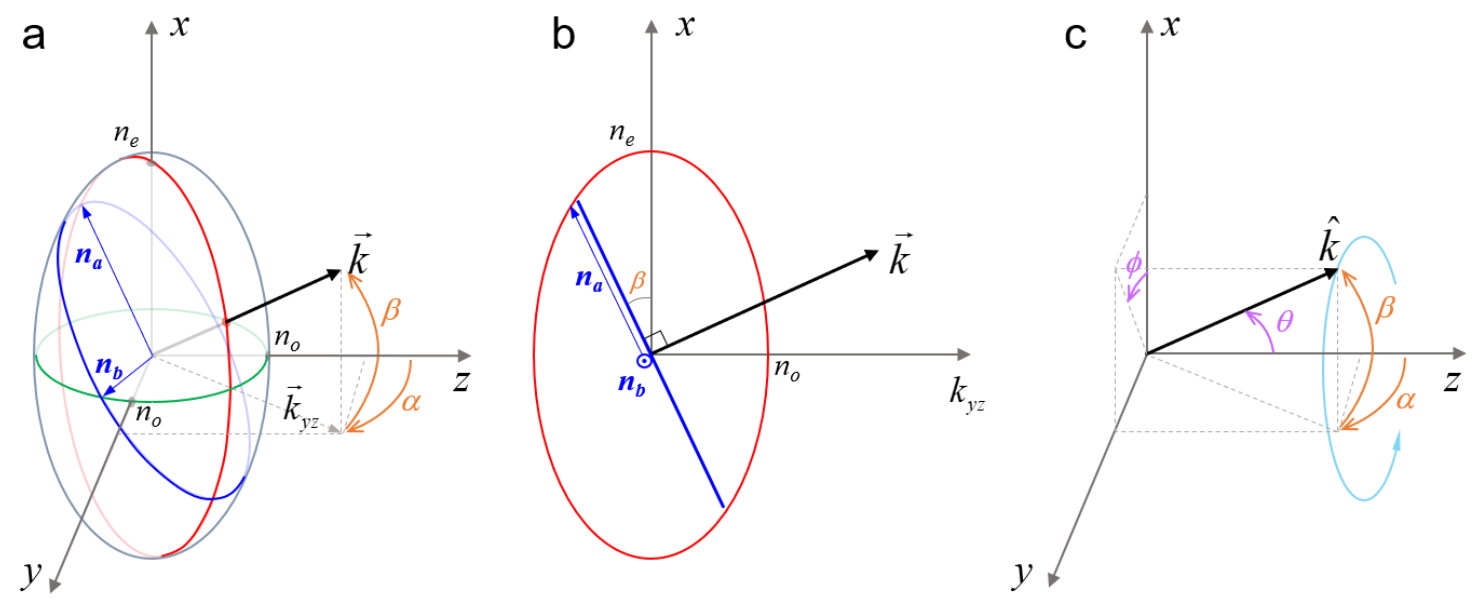

Fig. S2. Two normal mode refractive indices in the case of a uniaxial material. (a) A situation of an oblique illumination with wavevector $\vec{k}$. The refractive index ellipsoid is an ellipsoid of revolution which is formed along the principal axes. The $z$-axis is the optical axis for the normal illumination and the direction of the illumination light has an azimuthal and elevation angles of $\alpha$ and $\beta$ with respect to the $z$-axis. The index ellipse is represented in blue color and the lengths of its semi-axes correspond to the normal mode refractive indices $n_{a}$ and $n_{b} . \vec{k}_{y z}$ is the projection of the wavevector to the $y-z$ plane, $n_{o}$ and $n_{e}$ are the ordinary and extraordinary refractive indices of the uniaxial material. (b) The index ellipse observed along the direction of $n_{b}$ in (a). The ellipse is also drawn in red color in (a). Due to the symmetry along the rotation of $\alpha, n_{b}=n_{o}$, and $n_{a}$ is determined by the equation of the ellipse in Eq. (S8). The horizonal axis is the direction of $\vec{k}_{y z}$ in (a). (c) Coordinate for the angle scanning. Due to the spherical symmetry of the angular scanning, using $\theta$ and $\phi$ is more convenient than $\alpha$ and $\beta$ for considering the effect of angle variation. $\hat{k}$ is the unit wavevector. 
in Fig. S2 (a). The other normal mode index $n_{a}$ is determined from an ellipsoid which is defined by the intersection of the ellipsoid of revolution and a plane containing wavevector $\vec{k}$ as represented in red color in Fig. S2 (a), and it is separately presented in Fig. S2 (b) with the horizontal axis along vector $\vec{k}_{y z}$, a projection of $\vec{k}$ to the $y$-z plane. Then the normal mode indices have the following relation,

$$
\frac{\cos ^{2} \beta}{n_{e}^{2}}+\frac{\sin ^{2} \beta}{n_{o}^{2}}=\frac{1}{n^{2}(\beta)},
$$

where $n_{a}=n(\beta)$ and $n_{b}=n_{o}$, the extraordinary and ordinary refractive indices, respectively.

As shown in Eq. (S8), the normal mode index $n_{a}$ strongly depends on the rotation angle $\beta$ in general situations. Considering biological specimens, due to the weak birefringent effect, the difference of the two principal indices can be considered as very small such that $n_{e}=n_{o}+\delta$, and $\delta<<n_{e}, n_{o}$. With this condition, Eq. (S8) can be approximated as

$$
\begin{aligned}
\frac{1}{n^{2}(\beta)} & =\frac{\cos ^{2} \beta}{\left(n_{o}+\delta\right)^{2}}+\frac{\sin ^{2} \beta}{n_{o}^{2}}=\frac{\cos ^{2} \beta}{n_{o}^{2}\left(1+\delta / n_{o}\right)^{2}}+\frac{\sin ^{2} \beta}{n_{o}^{2}} \\
& \approx \frac{\cos ^{2} \beta}{n_{o}^{2}}\left(1-\frac{2 \delta}{n_{o}}\right)+\frac{\sin ^{2} \beta}{n_{o}^{2}}=\frac{1}{n_{o}^{2}}\left(1-\frac{2 \delta}{n_{o}} \cos ^{2} \beta\right) .
\end{aligned}
$$

Thus, the normal mode index $n_{a}$ is approximately obtained as

$$
n_{a}=n(\beta)=n_{o}\left(1-\frac{2 \delta}{n_{o}} \cos ^{2} \beta\right)^{-\frac{1}{2}} \approx n_{o}+\delta \cos ^{2} \beta
$$

With this approximation, the angle-dependent birefringence $\Delta(\beta)$ is determined as

$$
\Delta n(\beta)=n_{a}-n_{b}=n(\beta)-n_{o}=\delta \cos ^{2} \beta .
$$

$\Delta n(\beta)$ in Eq. (S10) is the birefringence from which the light suffers while traveling through the medium with an elevation angle $\beta$ with respect to the optical axis.

When the elevation angle stays around 0 , i.e., the angle scanning is achieved within a small range, the birefringence $\Delta n \approx \delta$. Thus, the effect of angle scanning is negligible when measuring the 
polarization response of a weak birefringent sample. But, for high NA applications such as our demonstrations, a wide range of angle scanning is required. Then the angle dependence in Eq. (S10) cannot be neglectable.

To take the effect of the angle scanning into account, we change the coordinate of the angle from $\alpha$ and $\beta$ to $\theta$ and $\phi$ as shown in Fig. S2 (c). With this change, the unit wavevector is represented as

$$
\begin{aligned}
\hat{k} & =(\sin \beta, \cos \beta \sin \alpha, \cos \beta \cos \alpha) \\
& =(\sin \theta \cos \phi, \sin \theta \sin \phi, \cos \theta) .
\end{aligned}
$$

From the relation in Eq. (S11), $\cos ^{2} \beta=\sin ^{2} \theta \sin ^{2} \phi+\cos ^{2} \theta=1-\sin ^{2} \theta \cos ^{2} \phi$. The birefringence in Eq. (S10) is then rewritten as

$$
\Delta n(\theta, \phi)=\delta\left(1-\sin ^{2} \theta \cos ^{2} \phi\right)
$$

Since the angle scanning is performed over the range of $\theta:\left[0, \theta_{\max }\right]$ and $\phi:[0,2 \pi]$, our measurement provides an average value of $\Delta n(\theta, \phi)$ over the scanning ranges. The average value in the spherical coordinate is then obtained as

$$
\begin{aligned}
\langle\Delta n\rangle & =\frac{1}{2 \pi\left(1-\cos \theta_{\max }\right)} \int_{0}^{2 \pi} \int_{0}^{\theta_{\max }} \Delta n(\theta, \phi) \sin \theta d \theta d \phi \\
& =\frac{1}{2 \pi\left(1-\cos \theta_{\max }\right)} \int_{0}^{2 \pi} \int_{0}^{\theta_{\max }} \delta\left(1-\sin ^{2} \theta \cos ^{2} \phi\right) \sin \theta d \theta d \phi \\
& =\frac{\delta}{6}\left(\cos ^{2} \theta_{\max }+\cos \theta_{\max }+4\right) \equiv \delta \eta_{\Delta n}\left(\theta_{\max }\right),
\end{aligned}
$$

where $\langle\Delta n\rangle$ is an average value of the birefringence and $\theta_{\max }$ is the maximum scanning angle along the $\theta$-axis. In Eq. (S13), $\eta_{\Delta n}\left(\theta_{\max }\right)$ is the factor including the effect of scanning and it decreases with the increase of $\theta_{\max }$ as shown in Fig. S3 (a). Despite the general tendency, the degree of reduction is not significant up to our experimental parameter. In our experiment, usually we scan the illumination angle $\theta_{\max }$ up to about $32^{\circ}$ providing the average birefringence as $\langle\Delta n\rangle \approx 0.93 \delta$ as denoted with the vertical dashed line in Fig. S3 (a). Although the birefringence 
observed with our experimental configuration can be slightly smaller than the actual value, but the discrepancy might be comparable to the experimental error.

The phase retardation depends on not only the birefringence but also the path length that the light takes while traveling through the material. For spherical particles such as LDs in cells, the physical path length of the light stays almost the same during the angle scanning. Thus, the phase retardation is directly proportional to the average birefringence, i.e., $\varphi_{R}=k\langle\Delta n\rangle d$, where $k$ is the wavenumber and $d$ is the diameter of the spherical particle. But, for a sample with a slab geometry such as the birefringence target in Fig. 2 in the main text, the variation of the light path should be taken into account. When a slab of weak birefringence is placed along the $x-y$ plane in the coordinate system in Fig. S2 (c), the path length of the light is $l(\theta)=d / \cos \theta$ with the scanning angles of $\theta$ and $\phi$, where $d$ is the thickness of the slab. The phase retardation at the scanning angles is given by $\varphi_{R}(\theta, \phi)=k \Delta n(\theta, \phi) l(\theta)$. Thus, the phase retardation observed with the angle scanning is described by the average of $\varphi_{R}(\theta, \phi)$ over the scanning range of $\theta:\left[0, \theta_{\max }\right]$ and $\phi:[0,2 \pi]$ as

$$
\begin{aligned}
\left\langle\varphi_{R}\right\rangle & =\frac{k}{2 \pi\left(1-\cos \theta_{\max }\right)} \int_{0}^{2 \pi} \int_{0}^{\theta_{\max }} \Delta n(\theta, \phi) l(\theta) \sin \theta d \theta d \phi \\
& =\frac{k \delta d}{2 \pi\left(1-\cos \theta_{\max }\right)} \int_{0}^{2 \pi} \int_{0}^{\theta_{\max }} \frac{1-\sin ^{2} \theta \cos ^{2} \phi}{\cos \theta} \sin \theta d \theta d \phi \\
& =\varphi_{R}^{0} \frac{1-\cos 2 \theta_{\max }-4 \ln \left(\cos \theta_{\text {max }}\right)}{8\left(1-\cos \theta_{\text {max }}\right)} \equiv \varphi_{R}^{0} \eta_{\varphi_{R}}\left(\theta_{\text {max }}\right),
\end{aligned}
$$

where $\left\langle\varphi_{R}\right\rangle$ is the average phase retardation, $\varphi_{R}^{0}=k \delta d$ the phase retardation measured at normal incidence. In Eq. (S14), $\eta_{\varphi_{R}}\left(\theta_{\max }\right)$ is the factor including the scanning effect and its variation is shown in Fig. S3 (b). It slightly increases over $\theta_{\max }$, but its deviation from unity is very small providing 1.004 at $\theta_{\max }=32^{\circ}$ as denoted with the vertical dashed line in Fig. S3 (b). The deviation of the phase retardation from the actual value in the slab geometry is even slower since 

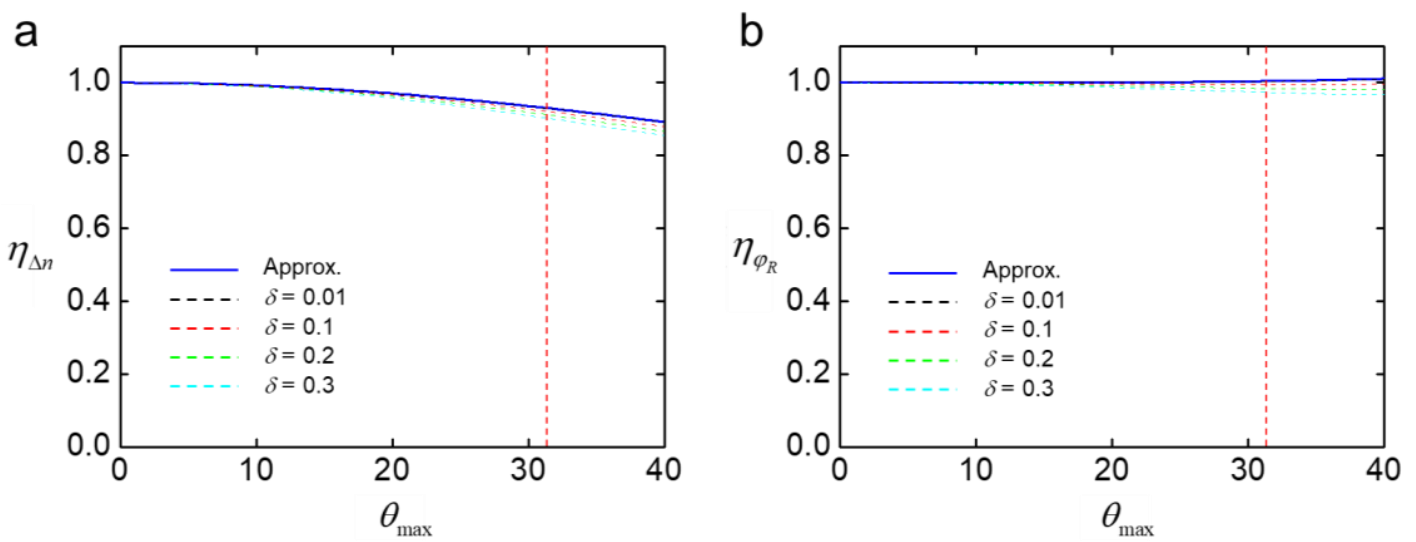

Fig. S3. The effect of angle scanning on a uniaxial material with weak birefringence. (a) $\eta_{\Delta n}$, the factor representing the effect of angle scanning in birefringence of spherical objects in Eq. (S13). (b) $\eta_{\varphi_{R}}$, the factor representing the effect of angle scanning in phase retardation in birefringence of a slab in Eq. (S14). $\theta_{\max }$ is the maximum scanning angle.

the reduction of the birefringence caused by the oblique illumination is compensated by the increase of the light path.

In Fig. 2 in the main text, the birefringent target has a slab geometry where the main source of the birefringence is the polymer structure posed as a uniaxial configuration. Its actual birefringence is known as $\delta=0.1555$ at $515 \mathrm{~nm}$ wavelength, then we can expect that the birefringence at $633 \mathrm{~nm}$ is not much different from that value. Thus, the assumption for the weak birefringence in Eq. (S9) and Eq. (S10) still holds and the result in Eq. (S14) is also valid for the birefringent target. For this reason, we can obtain the phase retardation quite close to the ground truth for the birefringent target in Fig. 2 in the main text.

\section{Biaxial Case:}

In a biaxial case where the principal indices $n_{1}, n_{2}$ and $n_{3}$ are all different, a birefringence along a specific direction can be obtained by the similar process to that for the uniaxial case. But due to the asymmetry of the index ellipsoid, not only the general description but also the approximation for the weak birefringence becomes even more complicated. Thus, here in this section, we discuss 
the biaxial cases for weak birefringence with the results of numerical simulations rather than the analytic approach.

We first set up an index ellipsoid with its principal axes $x_{1}, x_{2}$, and $x_{3}$ parallel to $x, y$, and $z$ axes of the coordinate, where the direction of $x_{1}$ coincides with $(1,0,0)$, as shown in Fig. S4 (a). For simulating a biaxial case, the principal indices were set as $n_{1}=n_{0}+0.2, n_{2}=n_{0}+0.1$, and $n_{3}=n_{0}$. The direction of the incident beam varied within a range of $\theta:\left[0, \theta_{\max }\right]$ and $\phi:[0,2 \pi]$ as we did in the experiments. The coordinates for $\theta$ and $\phi$ are the same as those in Fig. $\mathrm{S} 2$ (c). For every pair of $(\theta, \phi), \Delta n$ was numerically obtained and all the generated values were

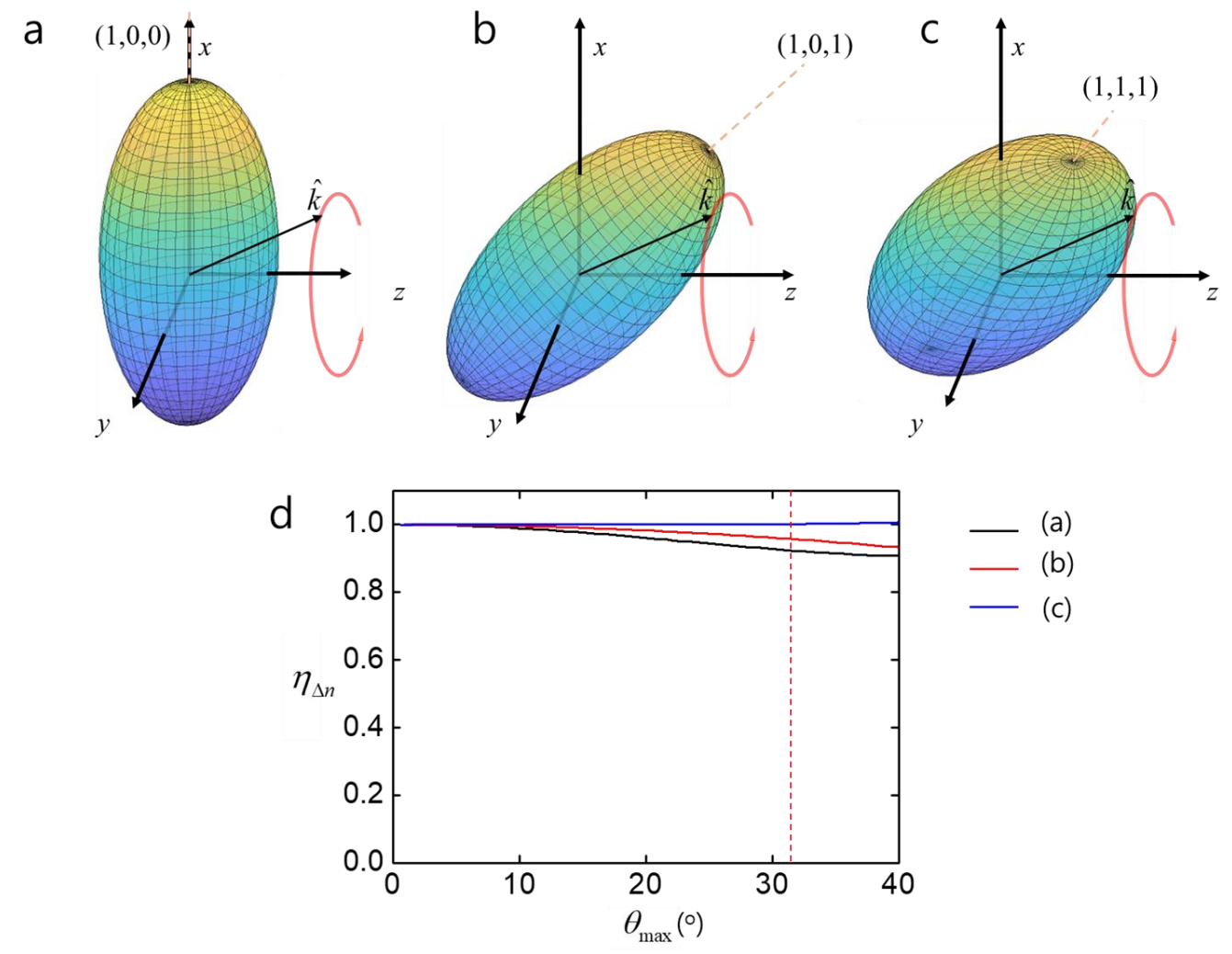

Fig. S4. Refractive index ellipsoids for biaxial cases. (a) The principal axes $x_{1}, x_{2}$, and $x_{3}$ coincide with $x, y$, and $z$ axes. (b) $x_{1}$ is parallel to $(1,0,1)$-direction with $x_{2}$ parallel to $y$-axis. (c) $x_{1}$ is parallel to $(1,1,1), x_{2}$ to $(0,1,-1), x_{3}$ to $(-1,1,1)$. For all cases, $n_{1}=n_{0}+0.2, n_{2}=n_{0}+0.1$, and $n_{3}=n_{0}$. The index ellipsoids are intentionally elongated for clear visibility. (d) $\eta_{\Delta n}$, the synthesized birefringence $\langle\Delta n\rangle$ normalized with that measured at normal incidence. Vertical dashed line indicates $\theta_{\max }=32^{\circ}$, the maximum scanning range in the experiments. 
averaged to determine $\langle\Delta n\rangle$. Then $\langle\Delta n\rangle$ was normalized by the birefringence value obtained at normal incidence along the direction of $(0,0,1)$ to attain $\eta_{\Delta n}$. This shows the relative deviation of $\langle\Delta n\rangle$ obtained by the synthetic process in our experiment from the value measured at normal incidence. Next, we rotated the index ellipsoid such that the direction of $x_{1}$ was parallel to the directions of $(1,0,1)$ and $(1,1,1)$ as shown in Figs. S4 (b) and (c), respectively. For each case, we repeated the similar calculation to obtain the relative deviation of the synthesized birefringence $\eta_{\Delta n}$ from the normal value.

The obtained $\eta_{\Delta n}$ slowly decreases with the increase of $\theta_{\max }$ as shown in Fig. S4 (d). The case in Fig. S4 (c) stays almost unchanged and that in Fig. S4 (a) shows the smallest value, but it remains larger than 0.9 up to 53 degrees for all cases. Particularly at our scanning range with $\theta_{\max }=32$ degrees, $\eta_{\Delta n}=0.92,0.96$, and 1.00 for the cases in (a), (b), and (c), in that order. We repeated the same calculations while keeping the same index parameters but with different rotations of the index ellipsoid, and then confirmed that $\eta_{\Delta n}$ always remained greater than 0.9 within our scanning range. Although Fig. S4 presents the results of biaxial cases with the specific index configurations, the simulation implies that under the weak birefringence condition the variation of the birefringence caused by the angular average stays small enough within the limited scanning angle so that the aperture synthesis produces the birefringence quite close to the value at normal incidence. Thus, in our approach, the aperture synthesis provides improved SNR with no significant uncertainty. 


\section{Supporting Information 4: Comparison of Jones matrix with Mueller matrix}

In this section, as a standard of conventional polarimetric imaging, we measured a Muller matrix of a sample and compared it with a Jones matrix. To this end, we placed multiple polarizers before and after a sample in the present Jones matrix microscopic system. Moreover, the optical configuration was designed to be easily switchable between the two imaging modes: Jones matrix and Mueller matrix imaging modes. In the Mueller matrix imaging, we measured 36 intensity images by alternating 6 different polarization states (horizontally linear, vertically linear, +45 degree linear, -45-degree linear, left circular, and right circular polarization states) on input and

a

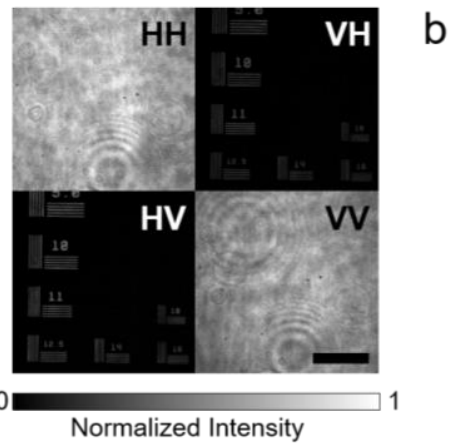

d

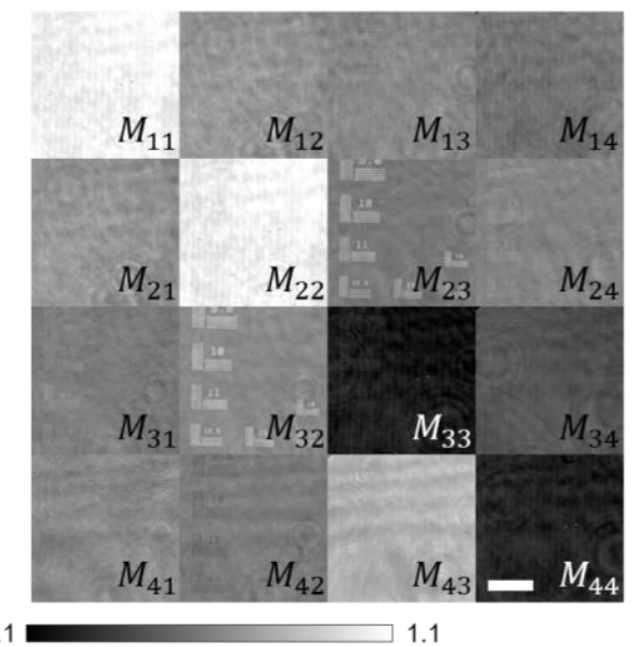

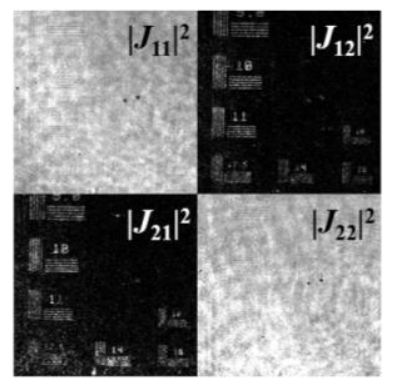

C

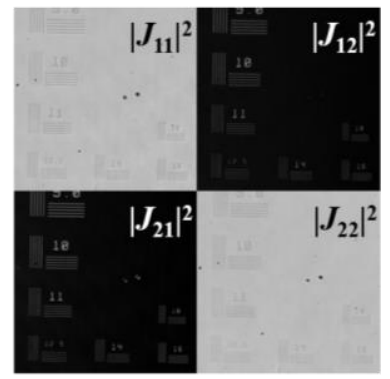

e

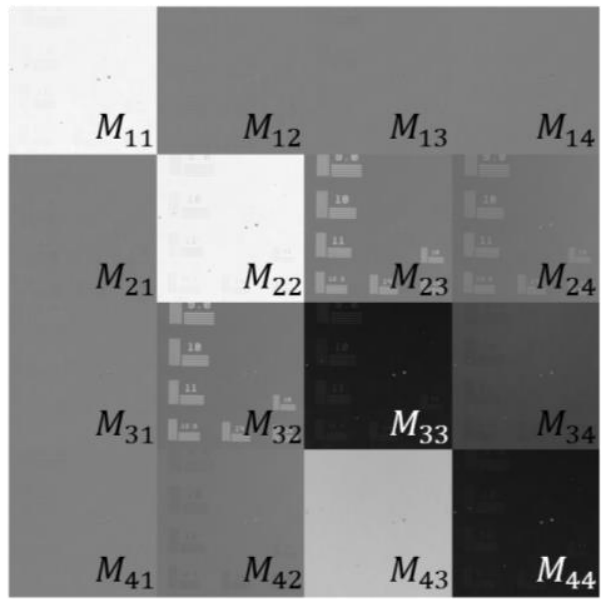

Fig. S5. Jones and Mueller matrix imaging of an NBS 1963A birefringent USAF target. (a) A set of polarized intensity images with various polarizer configurations. The first and second letters designate the axes of polarizers as $\mathrm{H}$ : horizontal polarizer and V: vertical polarizer. (b) Intensity maps (square of amplitude) of a single Jones matrix for the same target. (c) Same as (b) but synthesized. (d) The Mueller matrix of the target taken by the Mueller matrix imaging mode. (e) Mueller matrix obtained by transformation of the synthesized Jones matrix in (c). Scale bars: $2 \mathrm{~mm}$, colorbars: normalized intensity. 
output ports. The resultant Mueller matrix of a sample was calculated from the 36 polarized intensity images ${ }^{11,12}$.

For a comparative experiment, we used the NBS 1963A birefringent target with the 0 -degree optic axis was imaged with both the Jones and Mueller matrix imaging modes. Due to the polarizer configuration in the Jones matrix mode, the intensities of the Jones matrix elements describe the polarization alteration from one state to the other, specifically, $\left|J_{11}\right|^{2}$ for horizontal to horizontal, $\left|J_{12}\right|^{2}$ for vertical to horizontal, $\left|J_{21}\right|^{2}$ for horizontal to vertical, and $\left|J_{22}\right|^{2}$ for vertical to vertical. The intensity maps of the Jones matrix images were compared with the polarized intensity images measured using either the horizontally or vertically linear polarizers in the Mueller matrix imaging mode as shown in Figs. S5 (a)-(c). Figure S5 (a) shows the polarized intensity images taken by the Muller matrix imaging mode, where ' $\mathrm{H}$ ' and ' $\mathrm{V}$ ' in a pair mean 'horizontal' and 'vertical' polarizers located before and after the sample. Figures S5 (b) and S5 (c) present the intensity maps of the single and synthesized Jones matrices, respectively, and both the structural and background areas are similar to those in Fig. S5 (a). A relatively higher level of noise is found in both the polarized intensity image in Fig. S5 (a) and the single Jones matrix image in Fig. S5 (b), because of the long coherence length of the laser beam. In contrast, in the synthesized Jones matrix in Fig. S5 (c), the noise was significantly reduced and produced a better imaging quality than the prior two.

For further comparison, we measured the full 16 elements of the Muller matrix of the test target as shown in Fig. S5 (d). Next, the synthesized Jones matrix was transformed into a Muller matrix for the intuitive comparison, where we used the transformation formula ${ }^{13}$ from a Jones matrix to a Muller matrix expressed as

$$
M=A\left(J \otimes J^{*}\right) A^{-1}, A=\left[\begin{array}{cccc}
1 & 0 & 0 & 1 \\
-1 & 0 & 0 & 1 \\
0 & 1 & 1 & 0 \\
0 & i & -i & 0
\end{array}\right],
$$


where $M$ is a Mueller matrix, $A$ the transformation matrix, $A^{-1}$ the inverse matrix of $A, J$ the Jones matrix, and $\otimes$ denotes the Kronecker matrix product, and $*$ the complex conjugate. The transformed Muller matrix is presented in Fig. S5 (e). As seen in Figs. S5 (d) and (e), both the Muller matrices show almost the same intensity levels in all the elements. Thus it was verified that there was a good correspondence between the two measurements, one by the synthesized Jones matrix, the other by the conventional Muller matrix.

\section{Supporting Information 5: Polarization purity of a living cell}

We also measured the Muller matrix of a living cell, especially for the investigation of its depolarizing effect. Since the Jones matrix formalism represents only the coherent polarization states, the depolarized component cannot be handled once it occurs. Thus, we measured the degree of polarization caused by a living cell using the Muller matrix imaging mode. As a metric for the degree of polarization, we used two quantities: degree of polarization (DOP) and depolarization index (DI) ${ }^{14}$, both are commonly used for assessing the polarization purity of light and can be obtained from the measured Muller matrix.

Figure S6 (a) presents the Mueller matrix of a living HeLa cell taken by the Muller matrix imaging mode mentioned in Supporting Information 4. All the diagonal elements show a similar level of measured intensity. For the DOP, the output Stokes vectors with respect to the incidence Stokes vectors were obtained with either the horizontal or vertical linear polarization, the same as the input polarization states used in the Jones matrix measurement. With the output Stokes vector $\mathbf{S}=\left[\begin{array}{llll}\mathrm{S}_{0} & \mathrm{~S}_{1} & \mathrm{~S}_{2} & \mathrm{~S}_{3}\end{array}\right]^{T}$, the DOP map of the living cell can be obtained by using the formula, $\mathrm{DOP}=\sqrt{\mathrm{S}_{1}^{2}+\mathrm{S}_{2}^{2}+\mathrm{S}_{3}^{2}} / \mathrm{S}_{0}$ 
a

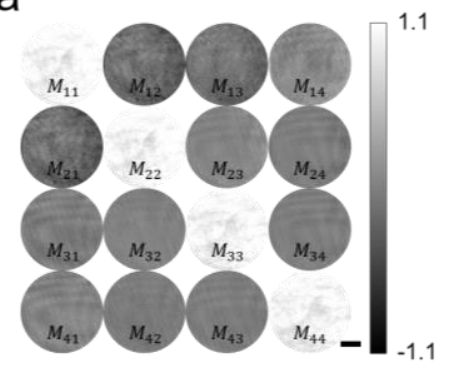

C

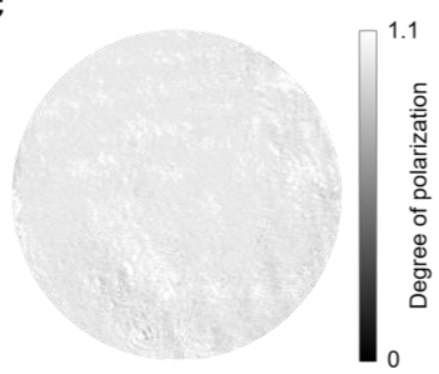

b

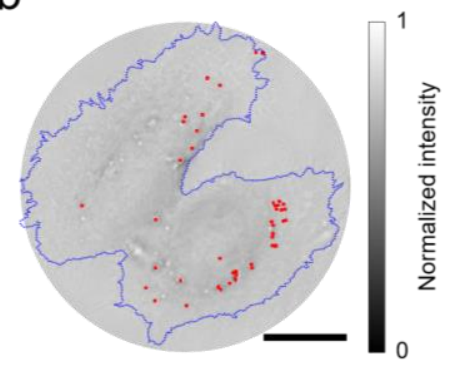

e

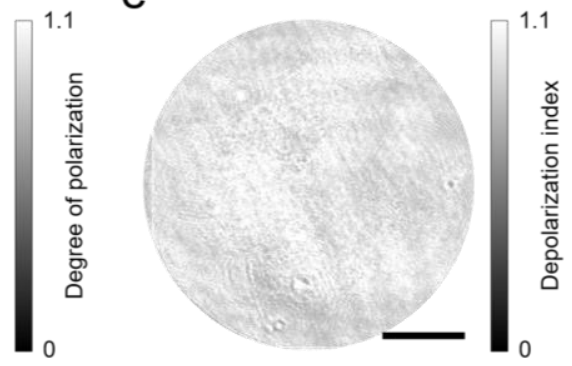

Fig. S6. Depolarizing effect by a living cell. (a) Mueller matrix of a living HeLa cell. (b) Whole cell area (blue) and LD region (red). (c) The DOP map measured with the horizontally linear polarized incidence wave. (d) The DOP map measured with the vertically linear polarized incidence wave. (e) The DI map. Scale bar: $10 \mu \mathrm{m}$.

We obtained the average of DOP values of the cell in two ways. One over the whole cell area including all LDs (lipid droplets) and the other only in the LD regions, as denoted with the blue and red lines in Fig. S6 (b) to see whether the LDs show different depolarizing characteristics. See Supporting Information 7 for more details about the determination of the LD region inside a cell. The average DOP value obtained in the whole cell area with the horizontally polarized incidence wave was measured as $1.01 \pm 0.05$, and that obtained only in the LD region was $1.00 \pm 0.04$. Since there is no difference in the two values, no distinguishable structures are found in the DOP map with a horizontally polarized incidence as shown in Fig. S6 (c). The average DOP value with vertically polarized incidence wave was measured as $1.01 \pm 0.02$ in the whole cell area and $1.01 \pm$ 0.02 in the LD region and the corresponding DOP map is shown in Fig. S6 (d). Again with the vertical polarization, there was no measurable difference between the cell area and LD region. Thus, from the DOP investigation, we could not find any sign of considerable depolarization occurring inside a living cell area including LD regions. 
We also investigated the DI values. The DI map can be obtained with the measured Mueller matrix $M$ by the following formula: $D I=\sqrt{\sum_{i, j=1}^{4} M_{i j}{ }^{2}-M_{11}{ }^{2}} /\left(\sqrt{3} M_{11}\right)$. Similar to the DOP case, the DI values were obtained both in the whole cell area and only in the LD regions. The DI value was measured as $0.99 \pm 0.10$ in the whole cell area and $0.97 \pm 0.10$ in the LD regions as shown in Fig. S6 (e), which shows almost no depolarization effect as the DOP maps.

From the Mueller matrix analysis by using the two parameters, it was confirmed that almost no depolarization effect occurs inside a living cell. Thus, the Jones matrix formalism can be applicable to the live cell measurements with no significant errors.

\section{Supporting Information 6: Comparison of polarization and fluorescence responses}

To confirm the hypothesis that the particles showing the strong polarization response in the cancer cells are LDs, we compared the phase retardation map of a cancer cell with its fluorescence image. For the fluorescence imaging, an epifluorescence microscope was additionally installed into the imaging part of the present optical system. A multi-channel collimated LED (Thorlabs, LED4D231) was used as an illumination source. The collimation beam was reflected off a dichroic beam splitter (DBS) and then illuminated on the sample for excitation. The emitted light transmitted through the DBS and was sent to the separate imaging path for the fluorescence measurement, which was newly built by using a flip mirror to minimize the disturbance to the existing polarization imaging path. The fluorescence image was captured by a CCD camera (Lumenera, Lt665RM). Overall magnification of the fluorescence imaging was designed as same as that for the polarization measurement. Accordingly, both the fluorescence and the polarization responses could be obtained from a stained sample by the switchable optical configuration. 
To visualize the intracellular LDs, sample staining was conducted with fluorophore Nile red (Invitrogen, N1142), which is widely used for the lipid, especially the LDs identification within cells ${ }^{15}$. Considering the Nile red has excitation/emission maxima near at $\sim 552 / 636 \mathrm{~nm}$, the LED channel (Center wavelength: $565 \mathrm{~nm}$ ) and a dichroic filter (Thorlabs, MD588) were chosen accordingly for the measurement.

For the preparation of multi-stained fluorescence imaging, cells on microscope slide were sufficiently washed with phosphate buffered saline (1× PBS; Gibco) buffer. For the intracellular lipid, the Nile red was diluted in a Rosewell Park Memorial Institute 1640 medium (RPMI; Hyclone) with a mixing ratio of 1:1000, and the diluted solution was added to the cells for 30 mins.

This treatment was done in a darkroom kept at room temperature. After that, the cells were washed with a PBS buffer. For the nucleic acid staining, Hoechst 33342 (Invitrogen, H3570) was diluted in PBS with a mixing ratio of 1:2000. The cells were treated with this solution for 20 mins. in an incubator and then washed with a PBS buffer. Finally, to make a microscope sample, a coverslip was placed on the slide and the edges of the coverslip were sealed to avoid medium leakage.
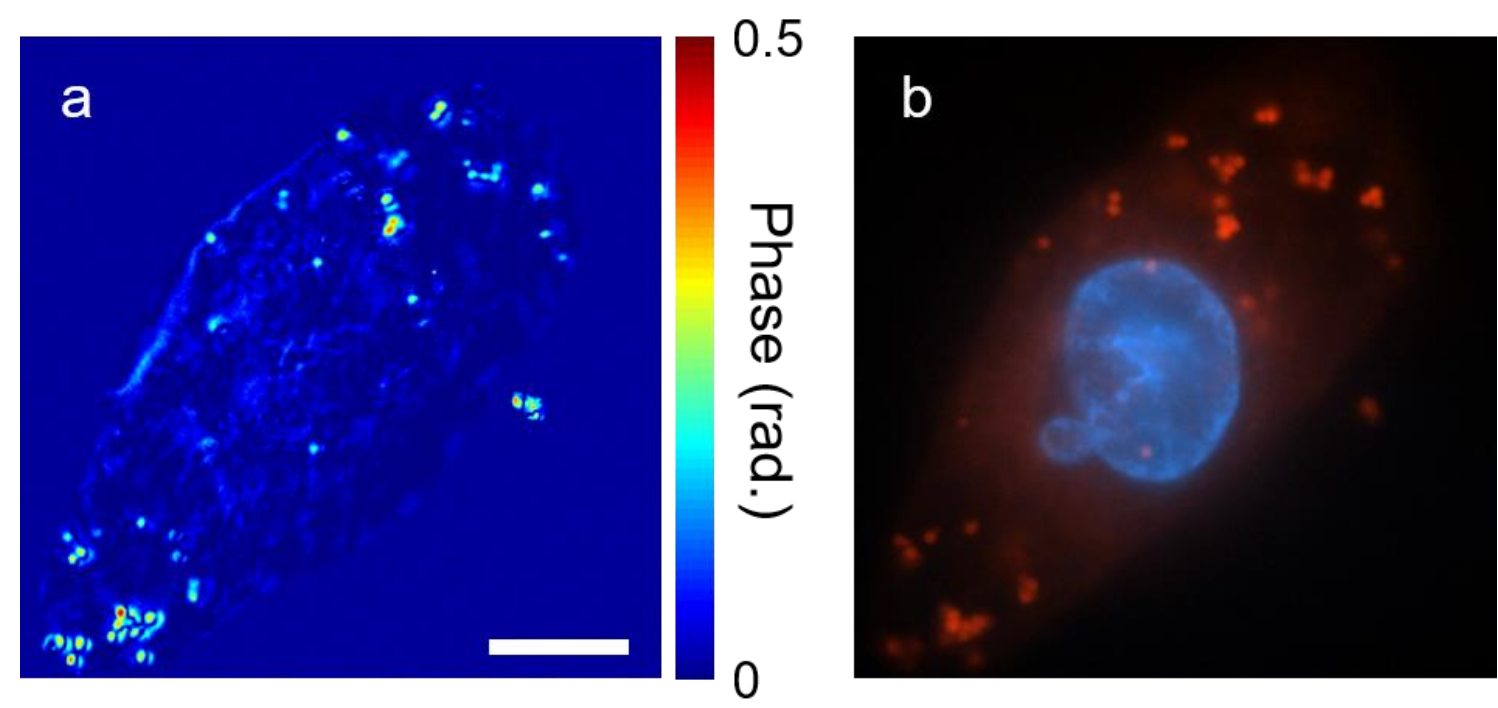

Fig. S7. Comparison of the phase retardation map with the fluorescence image of a H1650 cell. (a) A map of phase retardation. (b) The multi-color fluorescence image of the same cell stained with Nile red (red) and Hoechst 33342 (blue). Concentration of the Nile red and Hoechst 33342: $10 \mu \mathrm{g} / \mathrm{mL}, 5 \mu \mathrm{g} / \mathrm{mL}$, respectively. Scale bar: $10 \mu \mathrm{m}$. 
The taken phase retardation map and the fluorescence image are shown in Figs. S7 (a) and S7 (b), respectively. The distribution of the strong phase retardation in Fig. S7 (a) coincides with that of the LDs identified in red color in Fig. S7 (b). From this comparison, we concluded that the particles showing the strong polarization response in cells can be considered as LDs.

\section{Supporting Information 7: Construction of $P(x, y)$}

For the calculation of the total phase retardation, $R_{t o t}$, caused by small particles showing the strong polarization effect in cells, two images were employed to select only the particle regions. The phase retardation map (Fig. S8 (a)) was used with a certain threshold to determine the first binary mask shown in Fig. S8 (b). Since this mask has a certain noise around the particle due to the weak signal, we used 3-D tomogram to refine the mask. The 3-D tomogram of the cell can be reconstructed using the phase images taken during the Jones matrix measurement without any further acquisition.
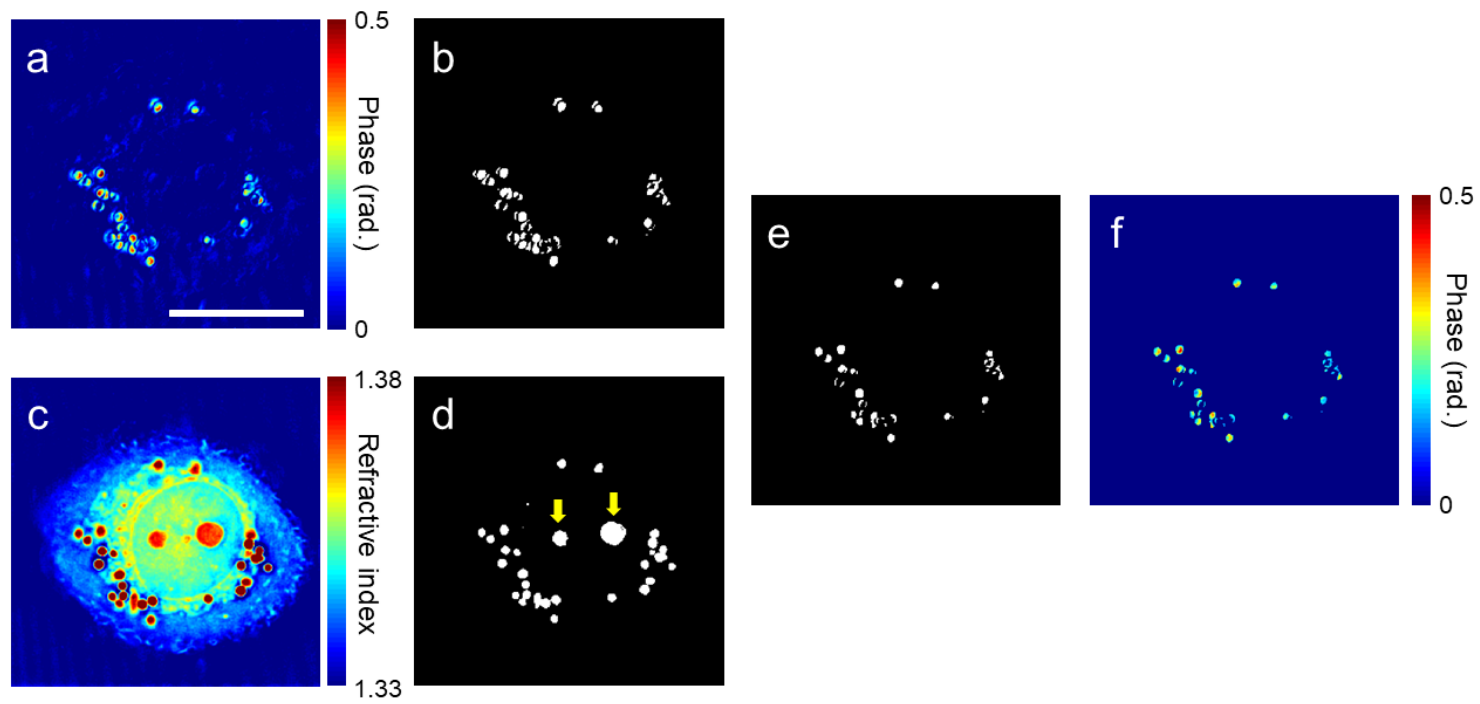

Fig. S8. Generation of the logical function. (a) $x-y$ tomogram of lung cancer cell (H1299) at focal plane.

(b) Logical image generated from (a). (c) Phase retardation map. (d) Logical image generated from (c).

(e) Phase retardation map containing only the selected region by the logical function. Scale bar:20 $\mu \mathrm{m}$. 
From the tomogram, the $x-y$ section at the focal plane $(z=0)$ could be extracted as shown in Fig. S8 (c). In the $x-y$ section image, the regions with higher refractive index than other area mostly coincide with those showing stronger phase retardation in Fig. S8 (a). Since the $x-y$ tomogram has better SNR than the phase retardation, it can be a good complement for choosing the regions with strong polarization responses. With a threshold value, another binary mask can be constructed as shown in Fig. S8 (d).

Although the tomogram provides a better SNR than the phase retardation map, but it also includes regions with non-polarizing effect. As indicated by yellow arrows in Fig. S8 (d), some regions are selected by the refractive index map, which is not chosen by the phase retardation map. These areas are inside the nucleus as seen in Fig. S8 (c). We found that these mismatches often arise particularly inside nuclear regions. Since it is very clear that these areas do not reveal any polarization responses in the phase retardation map, we eventually created the logical function $P(x, y)$ as a product of two binary masks determined by the phase retardation map and also the refractive index map. The result is shown in Fig. S8 (e). By applying the $P(x, y)$ to the phase retardation map in Fig. S8 (a), we finally produced the phase retardation map to determine $R_{t o t}$, where only the regions with the strong polarization were selected and others were made zero values as shown in Fig. S8 (f).

\section{Supporting Information 8: Geometrical effect in phase retardation of LDs}

The strong polarization responses of the LDs are thought to be mainly caused by lipid molecules, the main compound in LDs. However, since light polarization can be altered when crossing a curved interface, it is reasonable to expect that some portion of the strong polarization response is attributable to the shape of the LDs. 
To address this point, we investigated the degree to which the polarization effect can be caused by small spheres of various sizes. To evaluate the effect on the polarization response induced only by the sizes of the small particles, spheres made from polystyrene (PS), of which polarization change is known to be negligible, were used. From the 3-D tomograms of the cells, it was found that the sizes of the LDs seen in the cells ranged between from $471 \mathrm{~nm}$ to $991 \mathrm{~nm}$. Accordingly, the diameters of the PS beads were chosen to be in a similar range. Specifically, PS beads with sizes of $350,500,750$, and $1000 \mathrm{~nm}$ were used for the comparison measurements, and additional 3.28 $\mu \mathrm{m}$-diameter PS beads were used as a reference. To reduce the large refractive index contrast, the beads were immersed in refractive index-matching liquid $(n=1.56$, Cargille labs.) for the observation.

To directly compare the polarization responses of the PS beads with that of the LDs in the cancer cells, all the measured phase retardations are presented in the refractive index difference between the two axes, i.e., the birefringence. Figures S9 (a)-(d) show the birefringence images of the PS beads with sizes of 350, 500, 750, and $1000 \mathrm{~nm}$, respectively, and Fig. S9 (e) shows that with 3.28 $\mu \mathrm{m}$. A representative map of the birefringence in a cancer cell (H1299) is presented in Fig. S9 (f). As seen in the color scheme, the polarization response of the LDs in the cancer cell is much stronger than that of the PS beads.

For the direct comparison, the birefringence values taken from all LDs in the cancer cells and the that from the PS beads (10 for each) are presented with respect to their sizes in Fig. S9 (g). As seen in the figure, the LDs and PS beads show the same tendency, where the smaller the particle size, the greater the birefringence value. In Note, in Fig. S9 (g), we saw that the PS beads show a certain amount of birefringence which depends on their size although the medium of PS itself has negligible birefringence. Considering this point, the advent of noticeable birefringence in the PS beads can be attributed to the curvature of the particle surfaces. The spherical shape of the PS beads disturbed the polarization states of the incoming light. Owing to the larger curvatures of the smaller beads, they show stronger polarization alteration, while the larger beads reveal a smaller 
polarization effect. This was experimentally confirmed by using the $3.28 \mu \mathrm{m}$-diameter beads, of which size was sufficiently large compared to the wavelength of the light such that no measurable birefringence was found as shown in Figs. S9 (e) and S9 (g). Therefore, we can conclude that the birefringence shown by the PS beads was entirely caused by their geometric shapes.

In this measurement, the phase retardation $\varphi_{R}$ can be modeled as

$$
\varphi_{R}=\varphi_{N e t}+\varphi_{G}=\Delta n k d+\varphi_{G}
$$
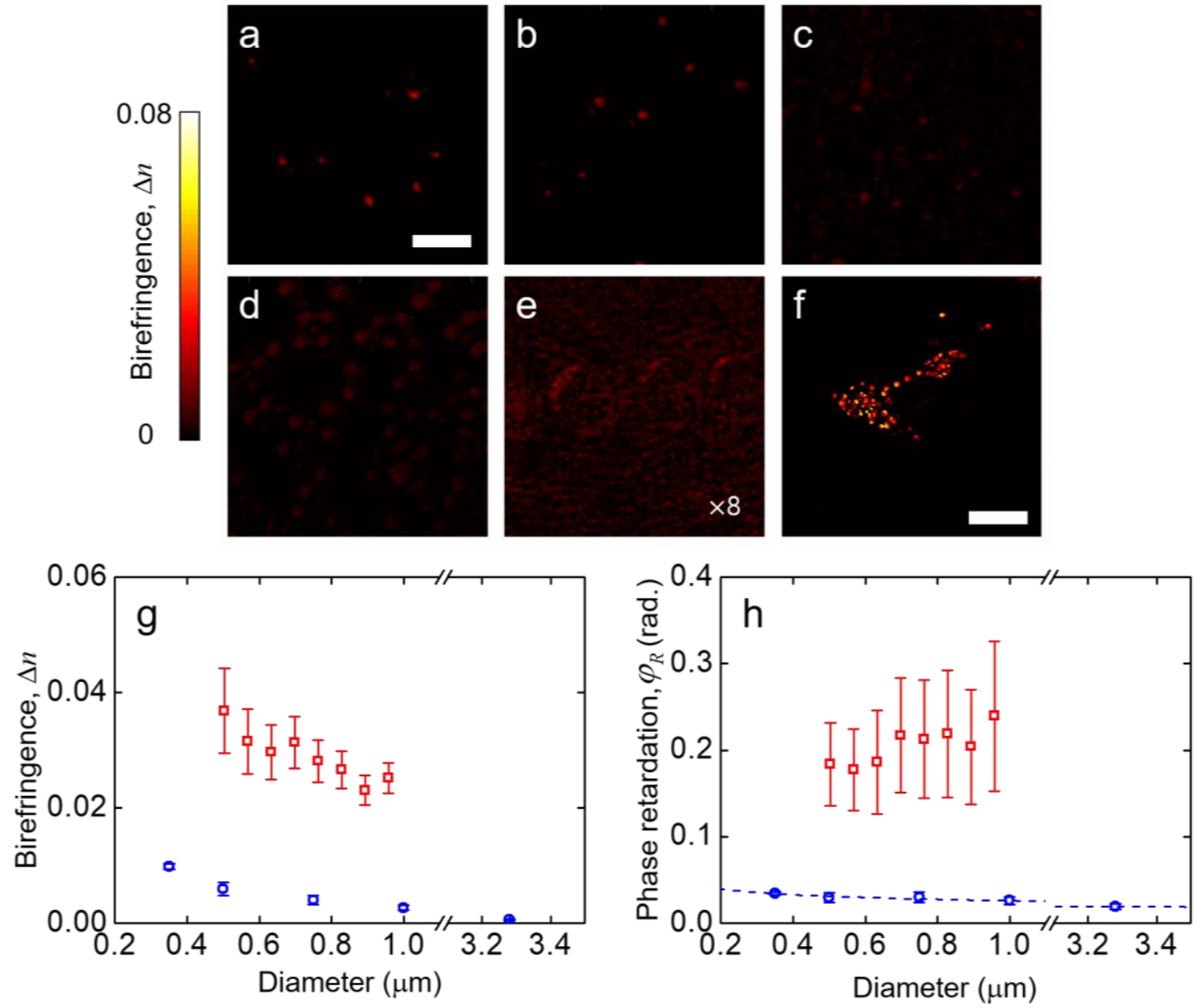

Fig. S9. Separation of the geometrical effect in birefringence of the LDs in cancers cells. (a-e) Birefringence maps of PS beads with diameters of (a) 350, (b) 500, (c) 750, (d) $1000 \mathrm{~nm}$, and (e) $3.28 \mu \mathrm{m}$. The color map in (e) is enhanced $8 \times$ for visibility. (f) Birefringence map of a lung cancer cell (H1299). (g) Average birefringence values of PS beads (blue) and LDs of all cancel cells (red). (h) Size dependence of the phase retardation measured in the PS beads (blue) and that in the LDs (red). Dashed line is a fitting. (a-e) Scale bar: $3 \mu \mathrm{m}$, (f) scale bar: $10 \mu \mathrm{m}$. 
In Eq. (S16), the total phase retardation $\varphi_{R}$ measured in the experiment is split into two terms, $\varphi_{N e t}=\Delta n k d$ and $\varphi_{G}$, where $\Delta n$ is the birefringence and $k=2 \pi / \lambda$, and $d$ the size of the object. The phase retardation caused by the inherent birefringence of the material composing the object is represented by $\varphi_{\text {Net }}$, and that by all the effects other than the birefringence is absorbed by $\varphi_{G}$. The main source of $\varphi_{G}$ is the curved interface, i.e., the shape of the object. Thus, $\varphi_{G}$, in general, depends on the size of the object with a spherical shape such as the PS beads and the LDs in our experiments because the curvature varies depending on the diameter of the objects.

We compared Eq. (S16) with the measurement results presented in phase retardation in Fig. S9 (h). Since $\Delta n_{P S} \approx 0$, for the PS beads, the measured phase retardation is all attributed to the object's shape, i.e., $\varphi_{R} \approx \varphi_{G}$. Although the actual dependence of $\varphi_{G}$ on $d$ is very complicated, but in our experimental range, it decreases very smoothly and monotonically as shown in Fig. S9 (h) and also in Fig. S10 (a). Thus, we used a simple fitting curve as

$$
\varphi_{G}(d)=a d^{-b}+c
$$
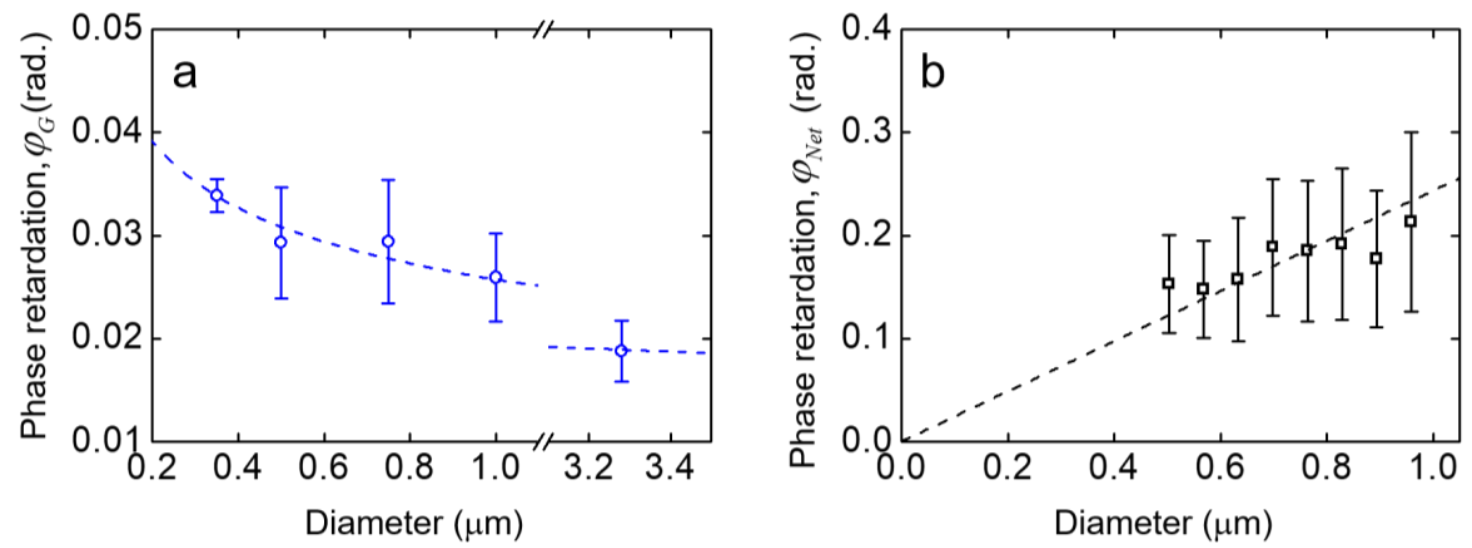

Fig. S10. Separation of the geometrical effect. (a) Phase retardation of PS beads. For the PS beads, $\varphi_{R}=\varphi_{G}$. Thus, the measured phase retardation $\varphi_{R}$ can be considered as $\varphi_{G}$. This graph is the same as that presented in blue in Fig. S4(h), but with different $y$-scale for better visibility. Dashed line is a fit curve by Eq. (S17). (b) $\varphi_{\text {Net }}$ for LDs after excluding $\varphi_{G}(d)$ from $\varphi_{R}$. Dashed line is a linear fit crossing the origin. 
Since the geometrical effect must vanish when $d$ becomes sufficiently large, then we used a constraint as $c=0$ when using the fitting curve. Consequently, by the fitting, $a$ and $b$ were determined as $(2.57 \pm 0.04) \times 10^{-2}$ and $(2.60 \pm 0.16) \times 10^{-1}$, respectively. The determined $\varphi_{G}(d)$ is presented in blue dashed line in Figs. S9 (h) and S10 (a).

Since the size effect was extracted clearly, the net phase retardation $\varphi_{\text {Net }}$ induced by the LDs could be extracted by subtracting $\varphi_{G}$ from that of the LDs (red squares in Fig. S9 (h)), i.e., $\varphi_{R}-\varphi_{G}$. The net phase retardation of the LDs in the lung cancer cells after excluding the geometrical effect is depicted in Fig. S10 (b).

Since $\varphi_{\text {Net }}$ is linearly proportional to $d$ without any offset constant as the relation $\varphi_{\text {Net }}=\Delta n k d$, we fitted $\varphi_{\text {Net }}$ with a straight line with only one fit parameter of $\Delta n$, where the slope corresponds to the birefringence of lipid, $\Delta n_{L P}$. By a linear fitting crossing the origin (black dashed line in Fig. S10 (b)), $\Delta n_{L P}$ for lipid was determined as $(2.45 \pm 0.12) \times 10^{-2}$. This net contribution can be attributed to the inherent property of the lipid and the value is very close to the birefringence of lipid compound reported elsewhere ${ }^{16-21}$. Thus, we experimentally confirmed that the strong polarization response of the LDs in cancer cells is indeed caused by the birefringence of lipid compound as well as the geometrical effect. 


\section{Supporting Information 9: $\boldsymbol{R}_{L D}$ of cancer subtypes}

In the main text, in Fig. 4, the difference of the total phase retardations $R_{L D}$ between the normal group $(N=20)$ and the cancer group $(N=103)$ was presented. It is shown in Fig. S11 (a) again just for the comparison. Even with the large variation on $R_{L D}$ values, the two groups were well separated by the polarization measurement.

The cancer group consisted of four subtypes as adenocarcinoma (AC), large cell carcinoma (LC), small cell carcinoma (SC1), and squamous carcinoma (SC2). We also compared $R_{L D}$ values among these cancer subtypes. The results are shown in Fig. S11 (b). The $R_{L D}$ values are (1.28 \pm $0.75) \times 10^{3}$ rad., $(1.31 \pm 1.00) \times 10^{3} \mathrm{rad} .,(1.83 \pm 1.32) \times 10^{3}$ rad., and $(2.42 \pm 1.96) \times 10^{3} \mathrm{rad}$. for AC $(N=25), \mathrm{LC}(N=31), \mathrm{SC} 1(N=26)$, and $\mathrm{SC} 2(N=21)$, in the order. Although the discrepancies among the mean values were identified, especially, the squamous cells showed significantly larger values than the others, but the statistically meaningful differences were not well recognized because of the large distributions of the $R_{L D}$ values.
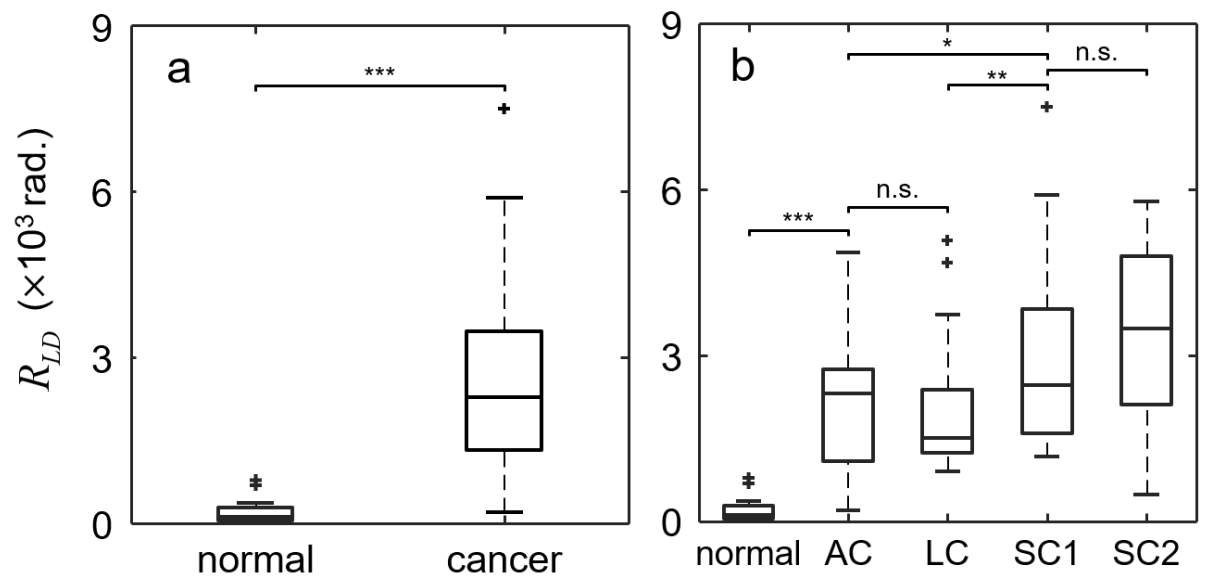

Fig. S11. Comparison of total phase retardation. (a) Total phase retardation for normal and cancer cells. (b) Box plot of $R_{L D}$ values for normal cells and cancer subtypes. Normal: normal cells $(N=20)$, AC: adenocarcinoma $(N=25)$, LC: large cell carcinoma $(N=31), \mathrm{SC} 1$ : small cell carcinoma $(N=26)$ and SC2: squamous cell carcinoma $(N=21)$. Asterisks present the Mann-Whitney $\mathrm{U}$ test: $* * *(P$-value $\leq$ $0.001)$, ** $(P$-value $\leq 0.01)$ and $*(P$-value $\leq 0.05)$. Symbol 'n.s.' means that the statistical difference between two groups is not significant. 


\section{References}

1. Yang, T. D.; Park, K.; Kang, Y. G.; Lee, K. J.; Kim, B.-M.; Choi, Y., Single-shot digital holographic microscopy for quantifying a spatially-resolved Jones matrix of biological specimens. Optics express 2016, 24 (25), 29302-29311.

2. Kim, Y.; Jeong, J.; Jang, J.; Kim, M. W.; Park, Y., Polarization holographic microscopy for extracting spatio-temporally resolved Jones matrix. Optics Express 2012, 20 (9), 99489955.

3. Liu, X.; Wang, B.-Y.; Guo, C.-S., One-step Jones matrix polarization holography for extraction of spatially resolved Jones matrix of polarization-sensitive materials. Optics letters 2014, 39 (21), 6170-6173.

4. Liu, X.; Yang, Y.; Han, L.; Guo, C.-S., Fiber-based lensless polarization holography for measuring Jones matrix parameters of polarization-sensitive materials. Optics express 2017, 25 (7), 7288-7299.

5. Shin, S.; Lee, K.; Yaqoob, Z.; So, P. T.; Park, Y., Reference-free polarization-sensitive quantitative phase imaging using single-point optical phase conjugation. Optics express 2018, 26 (21), 26858-26865.

6. Wang, Z.; Millet, L. J.; Gillette, M. U.; Popescu, G., Jones phase microscopy of transparent and anisotropic samples. Optics letters 2008, 33 (11), 1270-1272.

7. Choi, Y.; Kim, M.; Yoon, C.; Yang, T. D.; Lee, K. J.; Choi, W., Synthetic aperture microscopy for high resolution imaging through a turbid medium. Optics Letters 2011, 36 (21), 4263.

8. Kim, M.; Choi, Y.; Choi, W.; Fang-Yen, C. M.; Sung, Y.; Dasari, R. R.; Feld, M. S.; Kim, K., Three-dimensional differential interference contrast microscopy using synthetic aperture imaging. Journal of biomedical optics 2012, 17 (2), 026003.

9. Mico, V.; Zalevsky, Z.; García, J., Synthetic aperture microscopy using off-axis illumination and polarization coding. Optics communications 2007, 276 (2), 209-217.

10. Ralston, T. S.; Marks, D. L.; Carney, P. S.; Boppart, S. A., Interferometric synthetic aperture microscopy. Nature Physics 2007, 3 (2), 129.

11. Baba, J. S.; Chung, J.-R.; DeLaughter, A. H.; Cameron, B. D.; Cote, G. L., Development and calibration of an automated Mueller matrix polarization imaging system. Journal of biomedical optics 2002, 7 (3), 341-349.

12. Pezzaniti, J. L.; Chipman, R. A., Mueller matrix imaging polarimetry. Optical engineering 1995, 34 (6), 1558-1568.

13. Hunte, C., The Jones-Mueller transformation. FIZIKA A 2008, 17 (1), 51-58.

14. Chipman, R. A., Depolarization index and the average degree of polarization. Applied optics 2005, 44 (13), 2490-2495.

15. Pol, A.; Luetterforst, R.; Lindsay, M.; Heino, S.; Ikonen, E.; Parton, R. G., A caveolin dominant negative mutant associates with lipid bodies and induces intracellular cholesterol imbalance. The Journal of cell biology 2001, 152 (5), 1057-1070.

16. Jung, J.; Hong, S.-J.; Kim, H.-B.; Kim, G.; Lee, M.; Shin, S.; Lee, S.; Kim, D.J.; Lee, C.-G.; Park, Y., Label-free non-invasive quantitative measurement of lipid contents in individual microalgal cells using refractive index tomography. Scientific reports 2018, 8 (1), 1-10.

17. Mashaghi, A.; Swann, M.; Popplewell, J.; Textor, M.; Reimhult, E., Optical anisotropy of supported lipid structures probed by waveguide spectroscopy and its application to study of supported lipid bilayer formation kinetics. Analytical chemistry 2008, 80 (10), 3666-3676.

18. Minamikawa, T.; Ichimura-Shimizu, M.; Takanari, H.; Morimoto, Y.; Shiomi, R.; Tanioka, H.; Hase, E.; Yasui, T.; Tsuneyama, K., Molecular imaging analysis of microvesicular and macrovesicular lipid droplets in non-alcoholic fatty liver disease by Raman microscopy. Scientific reports 2020, 10 (1), 1-9. 
19. Ohshima, H., Encyclopedia of Biocolloid and Biointerface Science, 2 Volume Set. John Wiley \& Sons: 2016; Vol. 2.

20. Salamon, Z.; Tollin, G., Optical anisotropy in lipid bilayer membranes: coupled plasmonwaveguide resonance measurements of molecular orientation, polarizability, and shape. Biophysical journal 2001, 80 (3), 1557-1567.

21. Wolman, M., Polarized light microscopy as a tool of diagnostic pathology. Journal of Histochemistry \& Cytochemistry 1975, 23 (1), 21-50. 